\title{
A multiscale morphological approach to local contrast enhancement
}

\author{
Susanta Mukhopadhyay, Bhabatosh Chanda* \\ Electronics and Communication Sciences Unit, Indian Statistical Institute, Calcutta 700035, India
}

Received 15 March 1999; received in revised form 2 November 1999

\begin{abstract}
A scheme for enhancing local contrast of raw images based on multiscale morphology is presented in this paper. The conventional theoretical concept of local contrast enhancement has been extended in the regime of mathematical morphology. The intensity values of the scale-specific features of the image extracted using multiscale tophat transformation are modified for achieving local contrast enhancement. Locally enhanced features are combined to reconstruct the final image. The proposed algorithm has been executed on a set of raw images for testing its efficacy and the result has been compared with that of other standard methods for getting idea about its relative performance. (C) 2000 Elsevier Science B.V. All rights reserved.
\end{abstract}

\section{Zusammenfassung}

In diesem Beitrag wird ein Verfahren zur Verbesserung des lokalen Kontrastes von Rohbildern, basierend auf multiskalarer Morphologie vorgestellt. Das konventionelle theoretische Konzept zur lokalen Kontrastverbesserung wurde durch mathematische Morphologie erweitert. Die durch multiskale Zylindertransformation extrahierten Intensitätswerte der skalenspezifischen Eigenschaften des Bildes werden zur lokalen Kontrastverbesserung modifiziert. Lokal verbesserte Eigenschaften werden zur Rekonstruktion des Ergebnisbildes kombiniert. Der vorgeschlagene Algorithmus wurde an einem Satz Rohbilder zum Test seiner Effiziens angewandt und das Resultat wurde mit denen anderer Standardverfahren verglichen, um einen Eindruck seiner relativen Leistung zu erhalten. (C) 2000 Elsevier Science B.V. All rights reserved.

\section{Résumé}

Nous présentons dans cet article un schéma de rehaussement du contraste local dans des images, reposant sur de la morphologie multi-échelle. Le concept théorique conventionne du rehaussement de contraste local a été étendu dans le régime de la morphologie mathématique. Les valeurs d'intensité des caractéristiques spécifiques de l'échelle dans l'image, extraites en utilisant la transformation du chapeau haut-de-forme multi-échelle, sont modifiées pour atteindre un rehaussement du contraste local. Less caractéristiques rehaussées localement sont combinées pour reconstruire l'image finale. L'algorithme proposé a été exécuté sur un ensemble d'images afin de tester sont efficacité et les résultats ont été

\footnotetext{
* Corresponding author.

E-mail address: chanda@isical.ac.in (B. Chanda).
} 
comparés avec ceux d'autres méthodes standard afin d'avoir une idée de ses performances relatives. (C) 2000 Elsevier Science B.V. All rights reserved.

Keywords: Mathematical morphology; Multiscale morphology; Morphological towers; Tophat transformation; Local contrast enhancement

\section{Introduction}

The formation of a digital image of an object or a scene involves several intermediate steps like capturing the image, digitization, transmission, etc. The visual quality and the information content of the image so formed therefore suffer at almost all the steps in its formation. The image as a whole is found to have undergone a degradation and is unable to present the exact replica of the object or the scene. For example, a poor illumination of the object or the scene to be imaged causes a low contrast in the image whereas, the camera or the medium may introduce noise in the image data. Such a degraded image cannot render itself successfully for subsequent processing toward image understanding and interpretation. The technique usually adopted for improving the quality of the degraded image is broadly termed as image enhancement. Image enhancement is an ad-hoc process $[27,10,14]$ for improving the quality of an image by which a degraded image is made to acquire a pre-requisite quality for subsequent application specific processing. An enhancement technique performing well in enhancing biomedical images may not be identically efficient in enhancing satellite images. Conventional image enhancement techniques are broadly classified into two categories viz. the spatial domain techniques and the frequency domain techniques.

One of the most common degradations in the recorded image is its poor contrast. The contrast of an image may roughly be defined as the difference between its highest and lowest intensity values. The problem of poor contrast in the degraded image is usually solved by histogram stretching $[11,27]$ or by histogram equalization technique $[27,10]$. There are few variations of histogram equalization technique, e.g. histogram modification $[8,9]$. The conventional histogram equalization technique treats the image globally. Since the image characteristics differ considerably from one region to another in the same image, it is reasonable to adopt a context-sensitive technique based on local contrast variation especially when the local histogram does not follow the global histogram. Dorst [4] adapted histogram stretching method over a neighborhood around the candidate pixel for local contrast stretching. While, various modifications [26,25,23] of histogram equalization (or modification) are suggested based on adapting the same over a subregion of the image. Contrast stretching methods using local statistics are also reported [16,17,24]. Attas et al. [1] have devised a variational approach to local contrast enhancement through a suitable optimization of some desirable characteristics of graylevel histogram of output image. Similar approach for constrained local histogram equalization has been adopted by Zhu et al. [31]. Boccignone and Picariello [2] have suggested a multiscale approach to contrast enhancement using a non-linear scale-space representation of image generated by anisotropic diffusion. Another multiscale contrast enhancement technique is developed by Toet [30] through non-linear pyramid recombination.

Mathematical morphology [20,28] is a relatively new approach to image processing and analysis. This approach is based on set-theoretic concepts of shape. In morphology objects present in an image are treated as sets. As the identification of objects and object features directly depend on their shape, mathematical morphology is becoming an obvious approach for various machine vision and recognition processes. Several machine vision hardware manufacturers have started including morphological processors. These machines include Golay logic processor [7], Leitz Texture Analysis System TAS [15], the CLIP processor arrays [5], and the Delft Image Processor DIP [18]. Initially morphology dealt with binary images only, and basic operation 
were dilation and erosion, also known as Minkowski addition and subtraction [22], respectively. Natural extension of morphologic transformations from binary image processing to gray scale processing using max and min operations is done by Sternberg [29] and Haralick et al. [13]. Concept of multiscale morphology is introduced by Serra [28] followed by Maragos [19] and many other researchers. Multiscale morphologic techniques are also used in various types of image processing problems. Floreby et al. [6] have developed a noise removal technique using morphological pyramid decomposition and modified reconstruction. Multiscale morphologic edge detector is suggested by Chanda et al. [3].

In this paper we present a local contrast enhancement technique using multiscale morphology. We use usual notations of digital image processing and mathematical morphology [12] in the following description. Section 2 gives a brief discussion on conventional local contrast enhancement methods based on local statistics and local histogram equalization. Mathematical morphology alongwith multiscale morphology and tophat transfomation have been discussed in Section 3. In Section 4.1 we have first presented the theoretical formulation of local contrast enhancement using multiscale morphology. Section 4.2 presents elaborately various steps of the implementational aspects of the proposed alogorithm. Section 5 gives a discussion on the result and its comparison with that of other well-known techniques. Finally, concluding remarks are cited in Section 6.

\section{Local contrast stretching}

A good ambience of light illuminating the object or scene to be imaged may give rise to exhaustive utilization of the entire dynamic range of grayscale in the image, but the contrast over a small region may be very poor. Second, a relatively smaller number of pixels in such areas are insufficient to have any significant influence on the computation of global transformation. So the conventional histogram stretching or histogram equalization technique fail to serve the purpose. Such images need local enhancement and the technique by which this can be achieved is called local contrast stretching.

A contrast stretching method using local statistics suggested by Lee $[16,17]$ amplifies the difference between graylevel $g(r, c)$ and mean graylevel $\bar{g}(r, c)$ over a predefined neighborhood surrounding the pixel $(r, c)$. Thus the modified graylevel $\tilde{g}(r, c)$ is given by

$\tilde{g}(r, c)=\bar{g}(r, c)+k[g(r, c)-\bar{g}(r, c)]$

where $k$ is a global amplification factor and is greater than one. In another approach Narendra and Fitch [24] have considered the amplification factor also be a function of $(r, c)$ based on the local graylevel statistics over the same neighborhood as is used to define the mean graylevel $\bar{g}(r, c)$. Suppose graylevel variance over that neighborhood is $\sigma^{2}(r, c)$. Then the factor $k(r, c)$ is defined as

$k(r, c)=\gamma \frac{\bar{g}}{\sigma^{2}(r, c)}, \quad 0<\gamma \leqslant 1$

where $\bar{g}$ is the global mean of image graylevel and $\gamma$ is a user defined parameter.

The histogram equalization technique may be adopted to enhance the local contrast of the image. In this method the intensity of each pixel is modified through a local histogram equalization over a small region of the image around that pixel [23-26].

However, this kind of transformation using only local graylevel statistics cannot distinguish between consistent variation in intensity over a region and the variation in intensity due to presence of a feature (bright or dark) within a region. So it may stretch contrast evenly in both the cases. As a result, undesired contrast intensification (in supposedly smooth region) takes place at some regions of the image, which may require further processing such as de-enhancement [23]. On the other hand, if local contrast is stretched based on the presence of spatial features, then this problem can be avoided completely. As mathematical morphology is an appropriate tool for dealing with spatial features or shapes, we intend to modify Eq. (1) in terms of mathematical morphological operators. 


\section{Multiscale mathematical morphology}

Mathematical Morphology is a powerful technique in the field of image processing and computer vision. In morphology, the objects in an image are considered as set of points and operations are defined between two sets: the object and the structuring element (SE) $[28,12]$. The shape and the size of SE is defined according to the purpose of the associated application. Basic morphological operations are erosion and dilation. Other operation like opening (closing) is sequential combination of erosion (dilation) and dilation (erosion). We adopt, here, function- and set-processing (FSP) system [19]. FSP dilation of a graylevel image $g(r, c)$ by a two-dimensional point set $B$ is defined as

$(g \oplus B)(r, c)=\max \{g(r-k, c-l) \mid(k, l) \in B\}$.

Similarly, FSP erosion of $f(x, y)$ by $B$ is defined as

$(g \ominus B)(r, c)=\min \{g(r+k, c+l) \mid(k, l) \in B\}$.

The shape of the structuring element $B$ plays a crucial role in extracting features or objects of given shape from the image. However, for a categorical extraction of features or objects from the image based on shape and size we must incorporate a second attribute to the structuring element which is its scale. A morphological operation with a scalable structuring element can extract features based not only on shape but also on size. Also features of identical shape but of different size are now treated

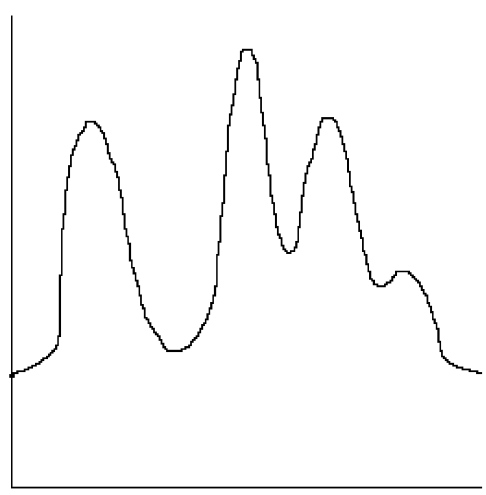

(a)

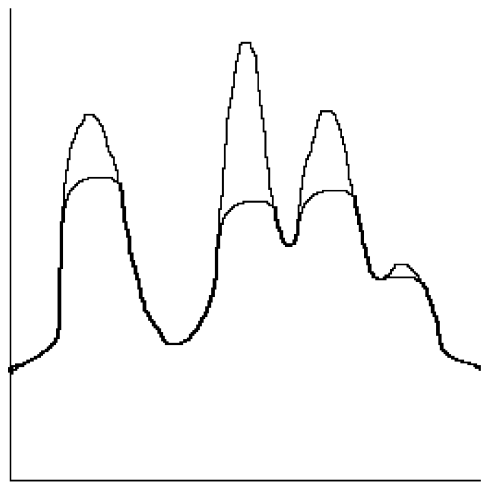

(c)

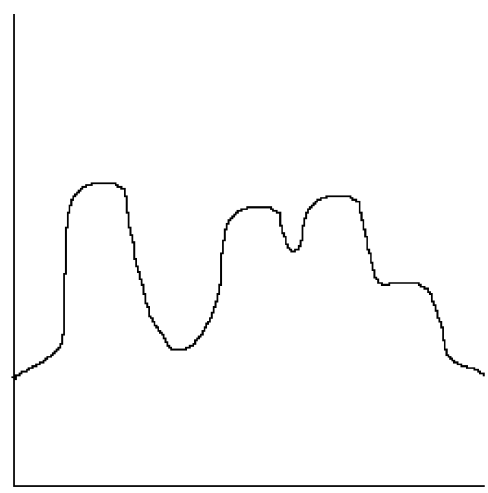

(b)

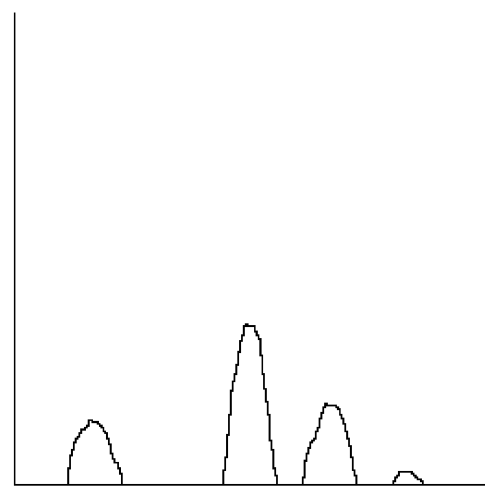

(d)

Fig. 1. Illustrating bright tophat transformation through graylevel opening: (a) original function, (b) function opened with circular disk, (c) superposition of the previous two and (d) features after tophat transform. 
separately. Such a scheme of morphological operations where a structuring element of varying scale is utilized is termed as multiscale morphology [28,19]. Multiscale opening and closing are defined, respectively, as

$(g \circ n B)(r, c)=((g \ominus n B) \oplus n B)(r, c)$,

$(g \bullet n B)(r, c)=((g \oplus n B) \ominus n B)(r, c)$,

where $n$ is an integer representing the scale factor of the structuring element. If $B$ is convex $n B$ is obtained by dilating $B$ recursively $n-1$ times with $B$ itself as [19]

$n B=B \underbrace{\oplus B \oplus B \oplus \cdots \oplus B}_{n-1 \text { times }}$.

By convention $n B=\{(0,0)\}$ when $n=0$.

\subsection{Multiscale tophat transformation}

The tophat transformation originally proposed in [21] provides an excellent tool for extracting bright (respectively, dark) features smaller than a given size from an uneven background. It relies on the fact that by gray-scale opening, one can remove from an image the brighter areas, i.e. features, that cannot hold the structuring element. Subtracting the opened image from the original one yields an image where the features that have been removed by opening clearly stand out. Similar thing is true for closing operation also. That means using a closing in stead of an opening and subtracting the original image from the closed one helps us extract dark features from a brighter background. Let us call it a black tophat transformation as opposed to white tophat transformation in case of opening. Suppose the structuring element used in both opening and closing is a disk, or, more specifically, a discrete approximation of disk. Therefore, the bright tophat tranformation decomposes an image into two parts as given by

$g(r, c)=\underbrace{(g \circ B)(r, c)}_{\text {part } 1}+\underbrace{[g(r, c)-(g \circ B)(r, c)]}_{\text {part } 2}$

where $B$ is a disk in discrete domain. Fig. 1 shows an example of bright tophat transformation for an one-dimensional signal. Let us call part 1 of Eq. (7) the base image with respect to $B(r, c)$. And let us call part 2 of Eq. (7) the feature image at size of $B$ as it contains all the bright features of $g(r, c)$ that are smaller than the size of $B$.

An ordered sequence of morphological tophat filtering through opening (closing) of the image with a disk structuring element at different scales extracts scale-specific bright(dark) features from the image. These scale-specific features resulting from the multiscale tophat transformation of the image can be amplified selectively to achieve local contrast stretching. Based on this notion we propose a local contrast enhancement method as described elaborately in the following section.

\section{Proposed method}

\subsection{Local contrast enhancement using morphology}

As mentioned in the previous section, the bright tophat tranformation decomposes an image into two parts. This may be expressed in terms of gray scale morphological operators as

$g(r, c)=\underbrace{(g \circ B)(r, c)}_{\text {part } 1}+\underbrace{[g(r, c)-(g \circ B)(r, c)]}_{\text {part } 2}$

where $(g \circ B)(r, c)$ is openning of graylevel image $g(r, c)$ by a graylevel structuring element $h(r, c)$ defined as

$h(r, c)=\left\{\begin{array}{cc}0 & \text { if }(r, c) \in B, \\ -\infty & \text { otherwise, }\end{array}\right.$

where $B$ is a disk in discrete domain. Then the graylevel structuring element $h(r, c)$ at scale $n$, in this case, may be defined as

$h(r, c)=\left\{\begin{array}{cl}0 & \text { if }(r, c) \in n B, \\ -\infty & \text { otherwise }\end{array}\right.$

The feature image [i.e. part 2 of Eq. (8)] gives a measure of local contrast in the original image due to presence of bright features. Hence, combining Eqs. (1) and (8) we suggest the following transformation for local contrast stretching:

$\tilde{g}(r, c)=(g \circ B)(r, c)+k[g(r, c)-(g \circ B)(r, c)]$

where $k$ is again a global amplification factor and is greater than one. So this transformation makes 
bright features brighter and, thus, improves the local contrast. Now suppose $k=2$. Eq. (9) becomes

$\tilde{g}(r, c)=g(r, c)+[g(r, c)-(g \circ B)(r, c)]$.

Let us denote $[g(r, c)-(g \circ B)(r, c)]$ by $F_{B}^{o}(r, c)$, i.e. features of size less than that of $B$ obtained by opening (more specifically, by bright tophat transformation). Accordingly, we can rewrite Eq. (10) as

$\tilde{g}(r, c)=g(r, c)+F_{B}^{o}(r, c)$.

Similarly we denote the bright-feature image at scale $n$ by $F_{n B}^{o}(r, c)$ as it contains all the features of $g(r, c)$ that are smaller than $n B$. Hence, the value $F_{n B}^{o}(r, c)$ of bright-feature image at $(r, c)$ gives a measure of local contrast in the original image due to presence of bright features at scale $n$. Note that $F_{0 B}^{o}(r, c)$ is an all-zero image. Now, let us define

$\delta_{n}^{o}(r, c)=F_{n B}^{o}(r, c)-F_{(n-1) B}^{o}(r, c)$.

It is evident that $\delta_{n}^{o}(r, c)$ contains bright features of $g(r, c)$ that are larger than scale $(n-1)$, but smaller than scale $n$. Therefore, we obtain, using multiscale approach, local contrast stretching of bright features as

$$
\begin{aligned}
\tilde{g}(r, c)= & g(r, c)+k_{1} \delta_{1}^{o}(r, c) \\
& +k_{2} \delta_{2}^{o}(r, c)+k_{3} \delta_{3}^{o}(r, c)+\cdots
\end{aligned}
$$

where $k_{1}>k_{2}>k_{3}>\cdots$, since we know that smaller the size of a bright feature, more should be its intensity for detectibilty. Suppose the features upto the scale $m$ are needed to be enhanced then

$\tilde{g}(r, c)=g(r, c)+\sum_{i=1}^{m} k_{i} \delta_{i}^{o}(r, c)$.

Theoretically, $m$ may correspond to SE as large as the entire image; however, for all practical purpose $m$ is small. This is because large features, in general, contribute heavily to global histogram and thus can influence global contrast stretching in their favor. Secondly, the probability that $\delta_{i}^{o}(r, c)$ being null image is more as $i$ increases. So we ignore $\delta_{i}^{o}(r, c)$ for $i>m$. Now taking $k_{i-1}=k_{i}+1$ for all $i$ and choosing $k_{m}=1$ we finally have local contrast stretching of bright features as

$\tilde{g}(r, c)=g(r, c)+\sum_{i=1}^{m} F_{i B}^{o}(r, c)$.
Selecting $k_{m}=1$ we can avoid multiplication operation to compute enhanced image and, thus, the computational cost is reduced to some extent.

Proceeding in a similar way based on multi-scale dark tophat transformations we achieve local contrast stretching of dark features as

$\tilde{g}(r, c)=g(r, c)-\sum_{i=1}^{m} F_{i B}^{c}(r, c)$.

Hence, to obtain the modifed image, in which contrast of both (bright and dark) types of features are stretched locally, we add Eqs. (15) and (16) and divide the result by 2 to get

$$
\begin{gathered}
\tilde{g}(r, c)=g(r, c)+0.5 \sum_{i=1}^{m} F_{i B}^{o}(r, c) \\
-0.5 \sum_{i=1}^{m} F_{i B}^{c}(r, c) .
\end{gathered}
$$

The constant multiplier 0.5 is used for assigning equal or impartial weightages to both dark and bright features. For the sake of generalization, we slightly modify Eq. (17) as

$$
\begin{aligned}
\tilde{g}(r, c)= & g(r, c)+0.5 \sum_{i=n}^{m} F_{i B}^{o}(r, c) \\
& -0.5 \sum_{i=n}^{m} F_{i B}^{c}(r, c)
\end{aligned}
$$

where all the features, either dark of bright, smaller than scale $n$ are assumed to be noise in the image.

\subsection{Implementation}

The implementation of Eq. (18) describing feature based local contrast enhancement involves construction of a number of morphological towers as elaborated below.

\subsubsection{Construction of morphological towers}

The image to be enhanced is made to undergo a sequence of grayscale morphological opening operations with a disc structuring element and its higher-order homothetics. The resulting sequence of images are kept in a stack called the opening tower as shown in Fig. 2. An identical tower, called closing tower, is constructed with the sequence of the images resulting from multiscale closing of the 


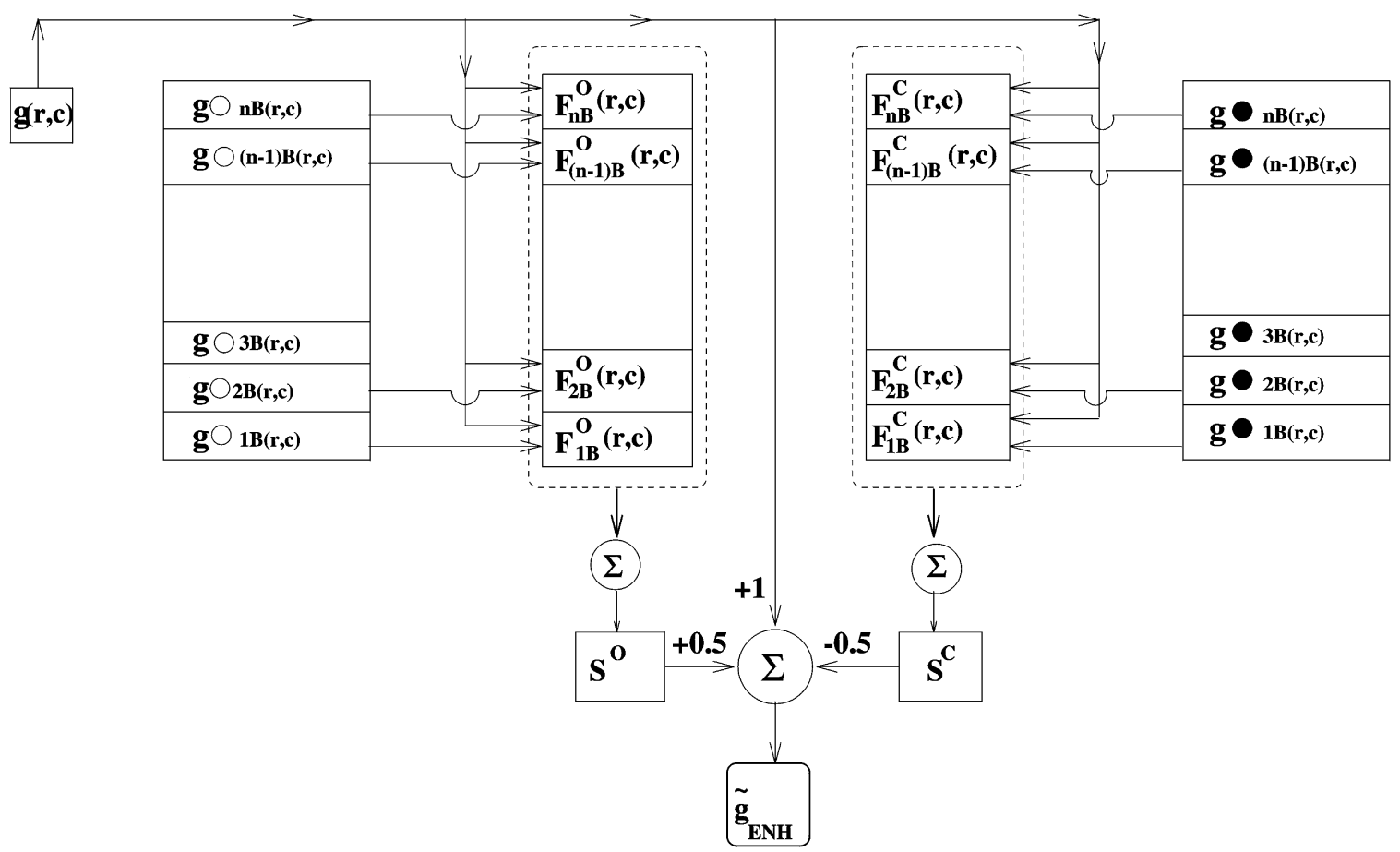

Fig. 2. Schematic diagram of different morphological towers.

input image. Therefore the $i$ th entry in the opening (closing) tower contains the image opened(closed) with the structuring element $i B$.

\subsubsection{Construction of difference towers}

The pixel values of opened image is less than or equal to that of the original image. Subtracting the opened image from the original one produces the feature image made up of bright features specific to the scale of the SE. Each entry of the opening tower is subtracted individually from the original image and the resulting bright feature images are then kept in corresponding entries in another tower called the difference tower. An identical difference tower is constructed for the dark feature images obtained by subtracting the original image from each entry of the closing tower. Therefore the $i$ th entry in the difference tower for opening (closing) contains an image consisting of bright (dark) features which are smaller than or equal to $i B$.

\subsubsection{Construction of the enhanced image}

For reconstructing the final image we do the following:

- We sum up all the entries in the difference tower corresponding to the opening operation. This results in an image consisting of bright features of all possible scales of interest that are present in the original image.

$S^{o}(r, c)=\sum_{i=1}^{n} F_{i B}^{o}(r, c)$.

The summation, here, denotes pixelwise sum of $n$ images.

- We perform the same operation on the difference tower corresponding to the closing operation. This results in an image consisting of dark features of all scales of interest that are present in the original image.

$$
S^{c}(r, c)=\sum_{i=1}^{n} F_{i B}^{c}(r, c) \text {. }
$$


Finally, the locally enhanced image is obtained by combining three images as given by

$\tilde{g}(r, c)=g(r, c)+0.5 S^{\mathrm{op}}(r, c)-0.5 S^{\mathrm{cl}}(r, c)$.

The '+' ' and '- ' operations are applied between corresponding pixels of three different images. However, it should be noted that at some pixels the value of $\tilde{g}(r, c)$ computed using Eq. (21) may exceed the allowed graylevel range. In that case the weights 0.5 may be replaced by $\alpha$ such that not more than $1 \%$ of total pixels are clipped.
The value of $\alpha$ can be selected between 0 and 0.5 using binary search technique.

\section{Experimental results and discussion}

The proposed algorithm has been tested on a set of biomedical images and the results have been compared with that of other methods [see Figs. 3-6]. Here we present detailed study with three example images. The images contain MR scan of

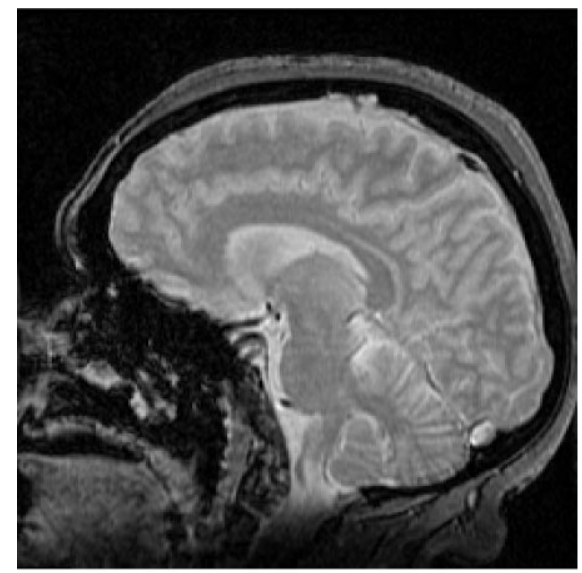

(a)

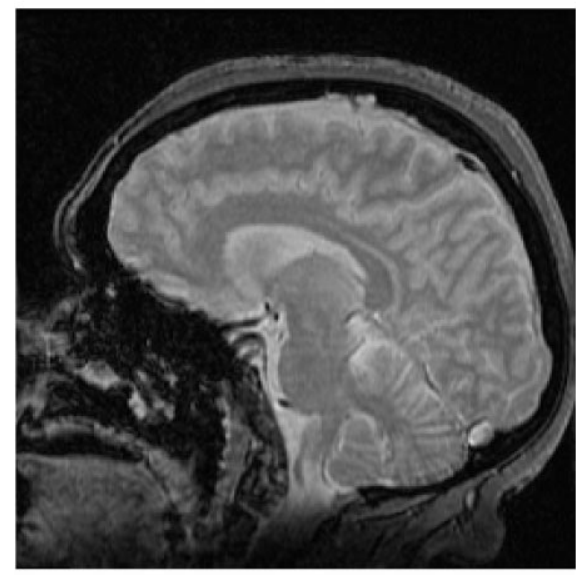

(c)

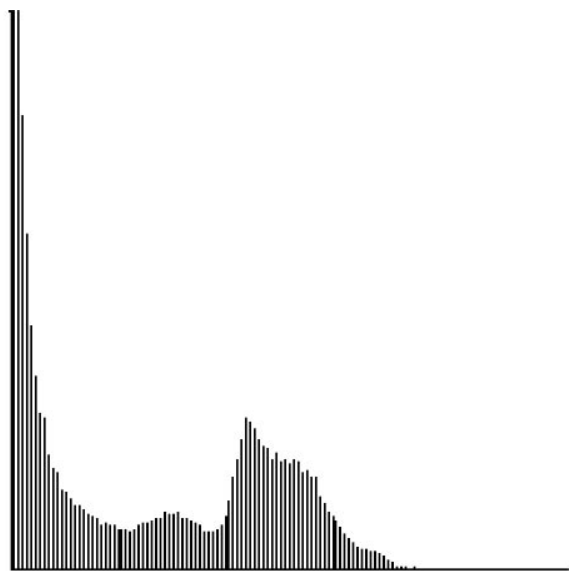

(b)

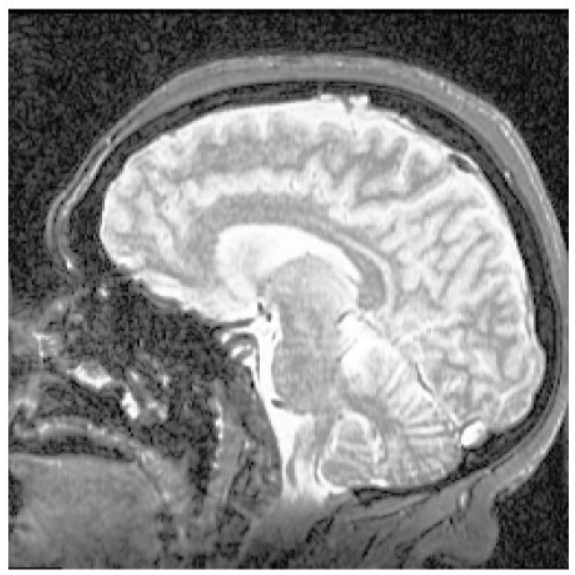

(d)

Fig. 3. (a) Original image, (b) histogram of the original image, (c) linear global contrast stretching and (d) global histogram equalization. 


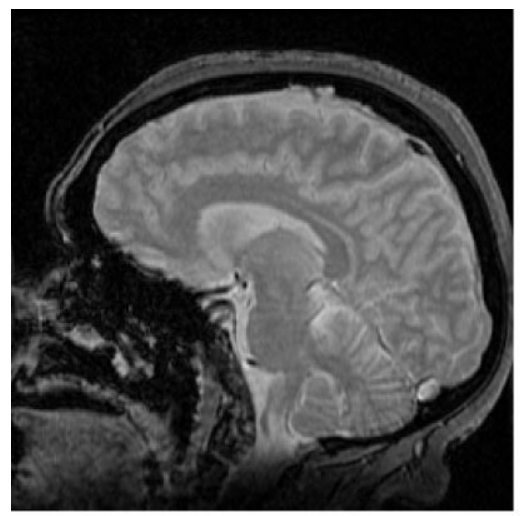

(a)

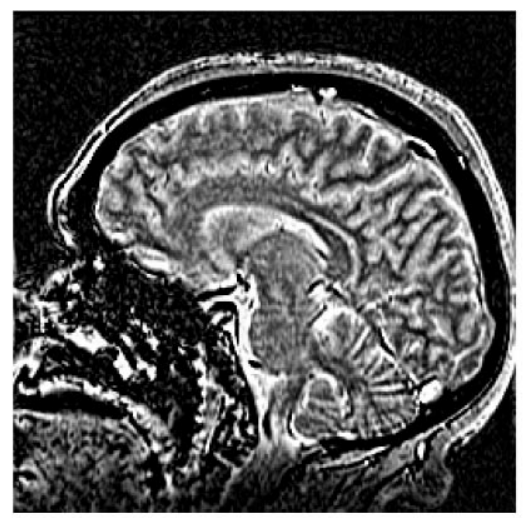

(b)

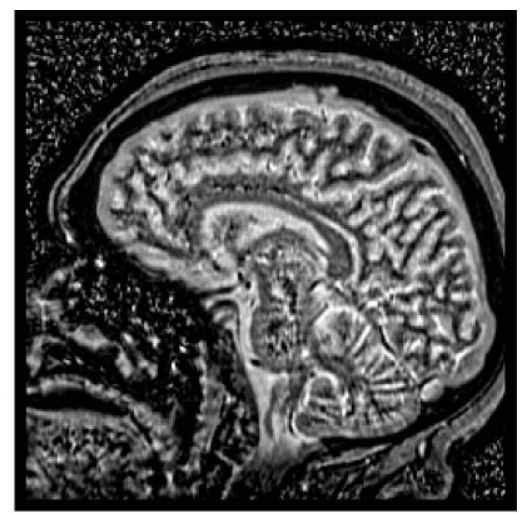

(c)

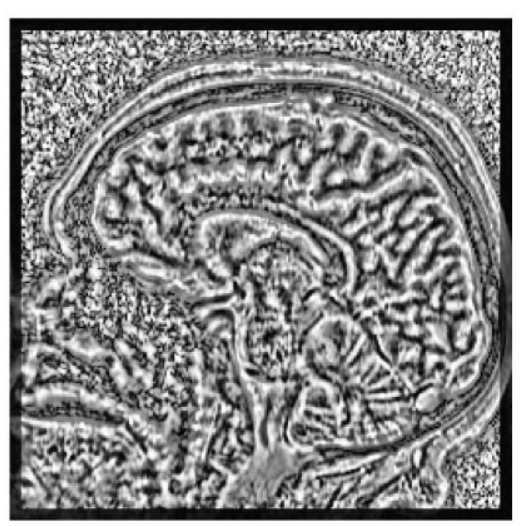

(d)

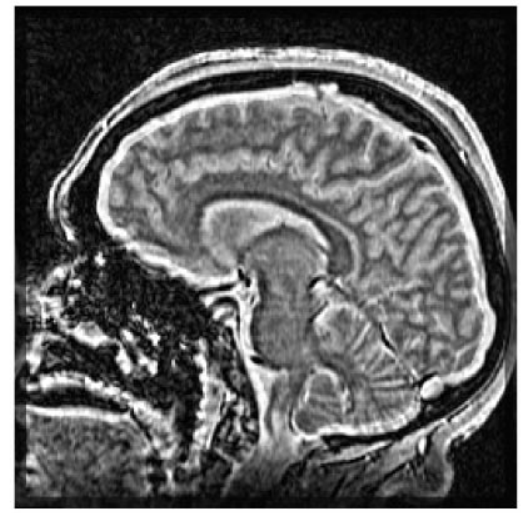

(e)

Fig. 4. (a) Original image, (b) local contrast stretching using multiscale morphology, (c) local contrast stretching based on local statistics [24], (d) local histogram equalization [23] and (e) local contrast stretching following the algorithm due to Dorst [4].

human brain. Fig. 3 shows the original image alongwith its graylevel histogram and the results of global enhancement techniques like linear contrast stretching and histogram equalization of it. The examples reveal that the global techniques cannot improve the contrast of the image satisfactorily. Results of the proposed algorithm are shown in Figs. 4-6 (b) where $n=1$ (as the images are assumed to be noise-free), $m=6$ and $B$ is a $3 \times 3 \mathrm{SE}$. This means largest SE used is $13 \times 13$. The value of $m$ can be made larger depending on demand or precision. However, it should be noted that very large value of $m$ will not give noticeable improvement in the output since the feature images of progressively larger scales have gradually smaller weightages in enhancing the image. Moreover, very large value of $m$, in spite of incorporating generality in the scheme, increases the computational time unnecessarily. The resulting image is seen to have more contrast than its original version. Results of other local contrast enhancement algorithms such as local histogram stretching [4], contrast amplification based on local statistics [24] and the local histogram equalization [23] are shown in Figs. 4c-e, respectively, so as to get some idea about their relative performance. In each case the size of the local window has been fixed at $13 \times 13$. The amplification factor in the algorithm due to Dorst [4] has been fixed at 3. For 


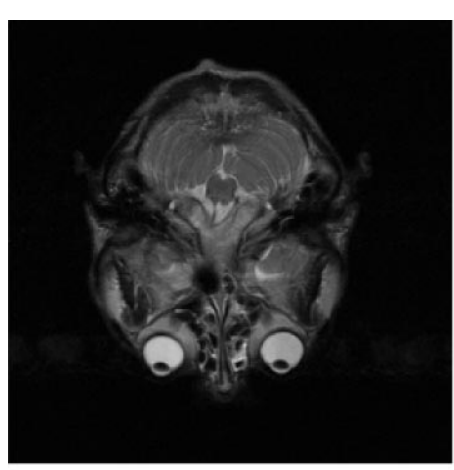

(a)

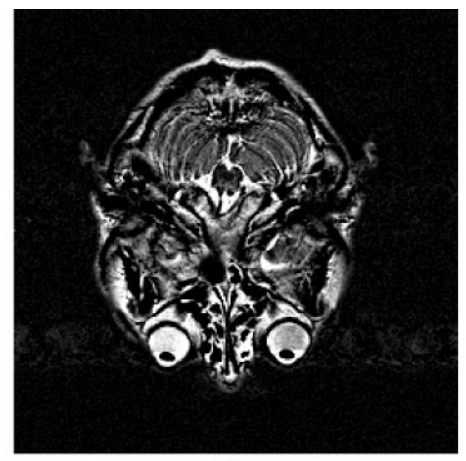

(b)

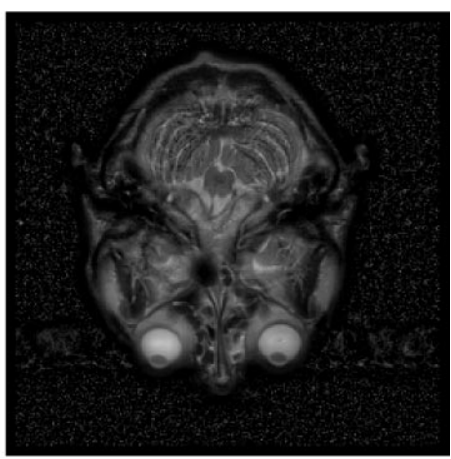

(c)

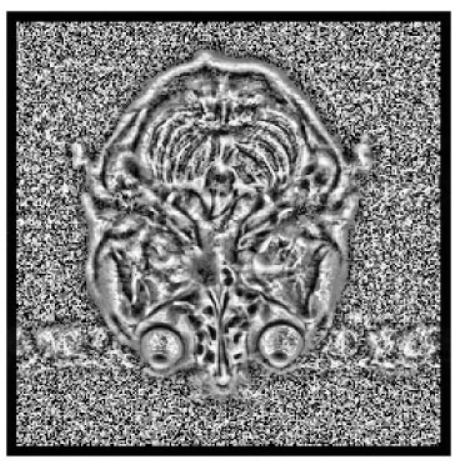

(d)

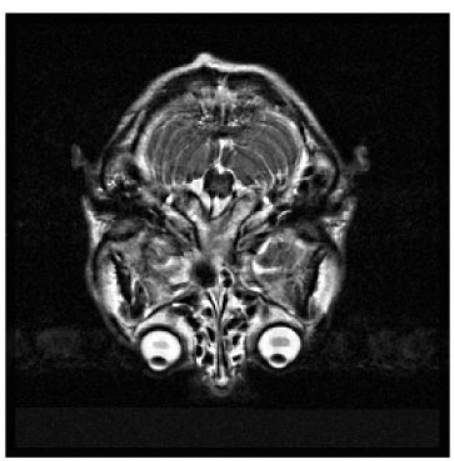

(e)

Fig. 5. (a) Original image, (b) local contrast stretching using multiscale morphology, (c) local contrast stretching based on local statistics [24], (d) local histogram equalization [23] and (e) local contrast stretching following the algorithm due to Dorst [4].

local contrast enhancement using local statistics due to Narendra and Fitch [24], the values for the minimum standard deviation and the scale factor chosen are 0.05 and 1, respectively. For local histogram equalization the threshold for the ratio of global mean to local variance is fixed at 0.5 . However, from the results, the enhancement of noise is found to be the least in case of morphological enhancement proposed by the authors. The significant local enhancement of the image without appreciable enhancement of noise proves the efficacy of the proposed method.

\section{Conclusion}

In this paper we have given a scheme for local contrast enhancement of graylevel images. The method employs multiscale morphological filtering in extracting the scale specific dark and bright features from the input image through the implementation of a number of towers. The recombination of all such features at the time of reconstruction emphasizes the basic requirement of local contrast enhancement which demands that features of progressively smaller scales should get more weights. The scheme has been implemented and executed on a set of gray-scale images. The results have been compared with those of few standard methods. The results due to the proposed method have been found reasonably satisfactory. However, the computational time and space required are high compared to other methods which the authors would like to improve subsequently. 


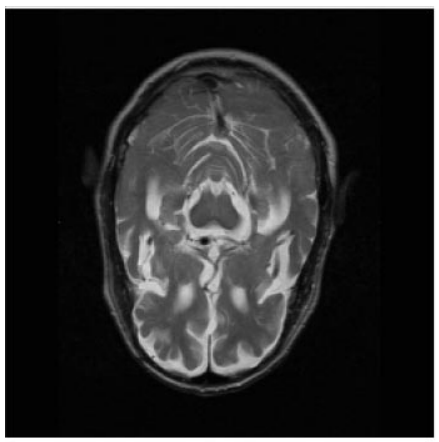

(a)

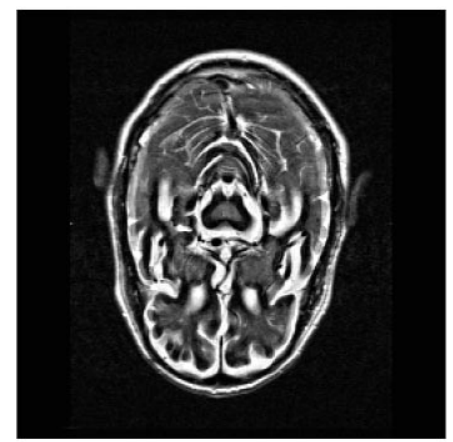

(b)

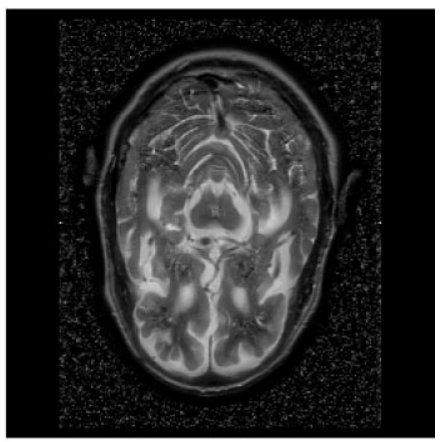

(c)

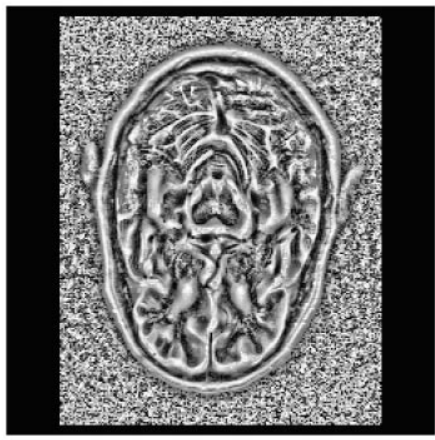

(d)

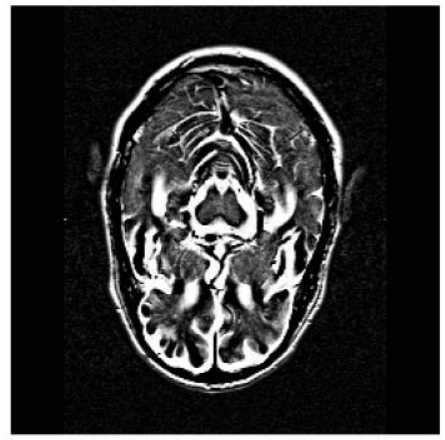

(e)

Fig. 6. (a) Original image, (b) local contrast stretching using multiscale morphology, (c) local contrast stretching based on local statistics [24], (d) local histogram equalization [23] and (e) local contrast stretching following the algorithm due to Dorst [4].

\section{References}

[1] I. Attas, J. Louis, J. Belward, A variational approach to the radiometric enhancement of digital imagery, IEEE Trans. Image Process. 4 (6) (June 1995) 845-849.

[2] G. Boccignone, A. Picariello, Multiscale contrast enhancement of medical images, Proceedings of the ICASSP'97, Vol. 4, Munich, Germany, April 21-24, 1997, p. 2789.

[3] B. Chanda, M.K. Kundu, Y.V. Padmaja, A multi-scale morphologic edge detector, Pattern Recognition 31 (10) (October 1998) 1469-1478.

[4] L. Dorst, A local contrast enhancement filter, Proceedings of the Sixth International Conference on Pattern Recognition, Munich, Germany, 1982, pp. 604-606.

[5] M. Duff, Parallel processors for digital image processing, in: P. Stucki (Ed.), Advances in Digital Image Processing, Plenum, New York, 1979.

[6] L. Floreby, F. Satter, G. Salomonsson, Image enhancement by morphological pyramid decomposition and modified reconstruction, Proceedings of the ICASSP'97, Vol. 4, Munich, Germany, April 21-24, 1997, pp. 2585-2588.

[7] M.J. Golay, Hexagonal parallel pattern transformations, IEEE Trans. Comput. C-18 (1969) 733-740.
[8] R.C. Gonzalez, B.A. Fittes, Gray-level transformations for interactive image enhancement, Proceedings of the Second Conference on Remotely Manned Systems, 1975, pp. 17-19.

[9] R.C. Gonzalez, B.A. Fittes, Gray-level transformations for interactive image enhancement, Mech. Mach. Theory 12 (1977) 111-122.

[10] R.C. Gonzalez, P. Wintz, Digital Image Processing, 2nd Edition. Addison-Wesley, Reading, MA, 1987.

[11] E.L. Hall, Computer Image Processing and Recognition, Academic Press, New York, 1979.

[12] R.M. Haralick, L.G. Shapiro, Computer and Robot Vision, Vol. 1, Addison-Wesley, Reading, MA, 1992.

[13] R.M. Haralick, S.R. Sternberg, X. Zhuang, Image analysis using mathematical morphology, IEEE Trans. Pattern Anal. Mach. Intell. PAMI-9 (4) (July 1987) 142-156.

[14] A.K. Jain, Fundamentals of Digital Image Processing, Prentice-Hall, Englewood Cliffs, NJ, 1989.

[15] J.C. Klein, J. Serra, The texure analyzer, J. Microscopy 95 (1977) 349-356.

[16] J.S. Lee, Digital image enhancement and noise filtering by use of local statistics, IEEE Trans. Pattern Anal. Mach. Intell. 2 (1980) 165. 
[17] J.S. Lee, Refined filtering of image noise using local statistics, Comput. Graphics Image Process. 15 (4) (April 1981) 380-389.

[18] P.F. Leonard, Pipeline architechture for real time machine vision, Proceedings of the IEEE Computer Society Workshop on Computer Architecture for Pattern Analysis and Image Database Management, 1985, pp. 502-505.

[19] P. Maragos, Pattern spectrum and multiscale shape representation, IEEE Trans. Pattern Anal. Mach. Intell. 11 (7) (July 1989) 701-716.

[20] G.M. Matheron, Random Sets and Integral in Geometry, Wiley, New York, 1975.

[21] F. Meyer, Contrast feature extraction, in: J.L. Chermant (Ed.), Quantitative Analysis of Microstructures in Material Sciences, Biology and Medicine, Riederer Verlag, Stuttgart, Germany, 1978.

[22] H. Minkowski, Volume and oberflache, Math. Ann. 57 (1903) 447-495.

[23] D. Mukherjee, B.N. Chatterji, Adaptive neighborhood extended contrast enhancement and its modification, Graphical Models Image Process. 57 (3) (May 1995) 254-265.

[24] P.M. Narendra, R.C. Fitch, Real-time adaptive contrast enhancement, IEEE Trans. Pattern Anal. Mach. Intell. 3 (6) (November 1981) 655-661.
[25] R.B. Paranjape, W.M. Morrow, R.M. Rangayyan, Adaptive-neighborhood histogram equalization for image enhancement, Graphical Models Image Process. 54 (3) (May 1992) 259-267.

[26] S.M. Pizer, E.P. Amburn, J.D. Austin, R. Cromartie, A. Geselowitz, T. Geer, B.H. Romeny, J.B. Zimmerman, K. Zuiderveld, Adaptive histogram modification and its variation, Comput. Vision Graphics Image Process. 39 (3) (September 1987) 355-368.

[27] A. Rosenfeld, A.C. Kak, Digital Picture Processing, 2nd Edition, Vol. 1 \& 2, Academic Press, New York, 1982.

[28] J. Serra, Image Analysis Using Mathematical Morphology, Academic Press, London, 1982.

[29] S.R. Sternberg, Gray scale morphology, Comput. Graphics Image Process. 35 (3) (September 1986) 333-355.

[30] A. Toet, Adaptive multi-scale contrast enhancement through non-linear pyramid recombination, Pattern Recognition Lett. 11 (11) (November 1990) 735-742.

[31] H. Zhu, F.H.Y. Chan, F.K. Lam, Image contrast enhancement by constrained local histogram equalization, Comput. Vision Image Understanding 73 (2) (February 1999) 281-290. 